\title{
A Quantitative Approach to Reductions in Secure Computation
}

\author{
Amos Beimel ${ }^{1}$ and Tal Malkin ${ }^{2}$ \\ 1 Department of Computer Science, Ben-Gurion University \\ beimel@cs.bgu.ac.il \\ 2 Department of Computer Science, Columbia University \\ tal@cs.columbia.edu
}

\begin{abstract}
Secure computation is one of the most fundamental cryptographic tasks. It is known that all functions can be computed securely in the information theoretic setting, given access to a black box for some complete function such as AND. However, without such a black box, not all functions can be securely computed. This gives rise to two types of functions, those that can be computed without a black box ("easy") and those that cannot ("hard"). However, no further distinction among the hard functions is made.

In this paper, we take a quantitative approach, associating with each function $f$ the minimal number of calls to the black box that are required for securely computing $f$. Such an approach was taken before, mostly in an ad-hoc manner, for specific functions $f$ of interest. We propose a systematic study, towards a general characterization of the hierarchy according to the number of black-box calls. This approach leads to a better understanding of the inherent complexity for securely computing a given function $f$. Furthermore, minimizing the number of calls to the black box can lead to more efficient protocols when the calls to the black box are replaced by a secure protocol.

We take a first step in this study, by considering the two-party, honestbut-curious, information-theoretic case. For this setting, we provide a complete characterization for deterministic protocols. We explore the hierarchy for randomized protocols as well, giving upper and lower bounds, and comparing it to the deterministic hierarchy. We show that for every Boolean function the largest gap between randomized and deterministic protocols is at most exponential, and there are functions which exhibit such a gap.
\end{abstract}

\section{Introduction}

The ability to compute functions securely is one of the most fundamental cryptographic tasks. Very roughly, two-party secure computation (on which we focus in this paper) involves two parties, Alice and Bob, who want to perform some computation on their inputs without leaking any additional information which does not follow from the intended output.

It is known (c.f. 4191022 ) that not all functions can be computed securely in the information-theoretic setting. However, Goldreich and Vainish [16] and 
Kilian 18 proved that every function can be computed securely in the information theoretic setting, given a black box that computes some complete function, such as Oblivious Transfer or the AND function. This type of a reduction is useful, because the security of the protocol is automatically maintained (computationally) when the black box is replaced by any computationally secure implementation of the function (such implementations exist under computational assumptions). 1 Moreover, such reductions provide a qualitative separation between "easy" functions that can be securely computed without calling the black box, and the "hard" functions which are the rest. Indeed, the notion of a reduction plays a central role at the heart of cryptographic foundations research (similarly to its central role in complexity theory). For example, black-box reductions between different cryptographic primitives were given in 6 611]12]197, 5[1321].

A long line of research has focused on studying, in various settings, which functions belong to the "easy" category above, and which are "hard", as well as studying which functions are complete (which in some cases turned out to be the same as all hard functions). In particular, these questions have been answered (with full characterization) for Boolean functions [10], in the two-party model [22]1/3, and completeness results appear in [19|21|3|20]. However, these works do not give rise to a hierarchy of different degrees of hardness, as they do not distinguish among the different functions that can be computed with a specific complete (say AND) black box.

Such a hierarchy exists (for the information-theoretic reduction setting), by a result of Beaver [2], showing that for all $k$, there are functions that can be securely computed with $k$ executions of the AND black box but cannot be computed with $k-1$ executions of the black box. We explore the hierarchy in this work.

OuR GOALs. In this paper, we propose to take a quantitative approach, classifying functions by how many calls to the black box are required to compute them securely. Minimizing the number of calls to the black box is especially desired as it can lead to more efficient protocols when the calls to the black box are replaced by a secure protocol. This problem was previously investigated in an ad-hoc manner, for specific functions of interest (e.g., different forms of OT). In most cases, only upper bounds on the number of calls were given. Two exceptions are Beaver [2] who proved that securely computing $n$ outputs of $\left(\begin{array}{l}2 \\ 1\end{array}\right)$ OT with unrelated inputs requires at least $n$ calls to $\left(\begin{array}{l}2 \\ 1\end{array}\right)$ OT, and Dodis and Micali [14 who proved that securely computing $\left(\begin{array}{l}n \\ 1\end{array}\right)$ OT requires at least $n-1$ calls to $\left(\begin{array}{l}2 \\ 1\end{array}\right)$ OT (see also [24]).

We propose a systematic study of the quantitative approach to reductions in secure computation, towards a deeper understanding of the inherent complexity of securely computing functions. In particular, focusing for the sake of presentation on the AND black box, we ask the following questions:

- Is there a well-defined rich hierarchy of functions based on how many ANDs are required to securely compute them?

${ }^{1}$ In this paper we consider the honest-but-curious model where modular composition is fairly straightforward. In the malicious model modular composition holds as well. See 8 for definitions and results on modular composition in the malicious model. 
- Given a function, can we give upper bounds on how many ANDs suffice to securely compute it? Can we give lower bounds?

- Can we give a combinatorial characterization of the functions with a certain minimal number of ANDs?

These problems are interesting in several settings. For the first problem, Beaver [2] provided a negative answer (the hierarchy collapses) in the computational setting, and a positive answer in the information theoretic setting, for randomized protocols (and for randomized functions, as well). Recently, Ishai et al. 17] proved that the hierarchy collapses in the random oracle model as well.

We note that by results of [16], lower bounds on the number of ANDs imply circuit lower bounds, meaning that it would be very hard to prove super-linear lower bounds in $n$ for functions of the form $f:\{0,1\}^{n} \times\{0,1\}^{n} \rightarrow\{0,1\}$. However, it would be very interesting to prove such linear lower bounds and to try to explore tighter connections with circuit complexity and communication complexity 2 of the functions.

OuR REsults. We start the investigation by studying the information-theoretic, two-party, honest-but-curious setting, where the output of the AND black box is received only by Alice. Unless otherwise noted, we also consider protocols with perfect correctness and security. For this setting we prove the following results: Deterministic Protocols. For deterministic protocols are we show:

- A complete combinatorial characterization of the minimal number of ANDs required to securely compute $f$ (the characterization is a recursive one, based on the truth-table of $f$ ).

For finite functions one can find the optimal protocol using our characterization. However, in general, our characterization does not lead to an efficient algorithm that determines how many ANDs are required to compute a function securely. This motivates the following results:

- A simple, explicit upper bound on the number of ANDs required for $f$. This upper bound may be exponential in the size of the input.

- For Boolean functions $f$ we prove that the above upper bound is tight by showing a matching lower bound. This implies that for some functions, an exponential number of ANDs is necessary.

Randomized Protocols. For randomized protocols are we show:

- An exponential gap using randomization: There are functions for which the number of ANDs required in a randomized protocol is exponentially smaller than the number of ANDs required in a deterministic protocol. We further exhibit a tradeoff between the number of random bits used and the number of ANDs required for one such example (Inner Product) where there is an exponential gap.

${ }^{2}$ Naor and Nissim 25 give some connections between the communication complexity of a function and the communication complexity for securely computing the function. However, translating them into our model, the number of ANDs is exponential in the communication complexity. 
- A lower bound: We prove a lower bound, depending on the function truthtable, on the number of ANDs required by any secure randomized protocol. Using this lower bound, we prove that for Boolean functions the gap cannot be super exponential: For any randomized protocol with $q$ ANDs, there is a deterministic protocol for the same function with at most $2^{q}$ ANDs.

- Gap already with 4 ANDS: There is a function that can be securely computed by a randomized protocol with 4 ANDs, however, every deterministic protocol securely computing it requires at least 6 ANDs.

- No gap with 1 AND: The functions that can be securely computed with one call to the AND black box are the same as in the deterministic case with one AND (for which an explicit characterization is given).

- Gap between perfect and non-perfect protocols: There are functions that require at least a linear (in the input length) number of ANDs for any perfect (randomized) protocol, but can be computed with $k$ ANDs (for any $k$ ), achieving a protocol with $1 / 2^{k}$ probability of error and statistical distance.

- Lower bound for non-perfect protocols: We show that the one-way randomized communication complexity in the shared-randomness model is a lower bound for the number of ANDs required by non-perfect protocols.

Extensions to Other Models and Complete Functions. As explained earlier, we choose the simplest model of secure computation to consider our quantitative approach. Some of our results carry over directly to other models, and some questions still remain open in the other models. We hope that our paper would be a starting point for further research which will clarify the situation in more complex models as multi-party protocols, and the protocols that are secure against malicious parties.

Specifically, only Alice gets the output of the function while Bob should not learn any information on the input of Alice. This one-sided model is the correct model when considering malicious two-party secure computation where the first party to get the output can quit the protocol preventing the other party from getting the output. In the honest-but-curious model, the one-sidedness of the output is not the only possibility; we choose it since we want the simplest model. Some results on the two-sided model, where Alice gets an output $f$ Alice and Bob gets an output $f^{\mathrm{Bob}}$, appear in the full version of this paper.

Furthermore, we state all our results counting the number of ANDs needed. However, every finite function (a function with a constant number of inputs) can be computed securely using a constant number of ANDs, and the AND function can be computed with one call to any complete function (this is implied by results of [3]). So, the results of this paper carry to every finite complete function, up to a constant factor. For example, the $\left(\begin{array}{l}2 \\ 1\end{array}\right)$ OT function can be computed securely with two ANDs. Thus, all lower bounds on the number of ANDs translate into the same lower bounds on the number of $\left(\begin{array}{l}2 \\ 1\end{array}\right)$ OT up-to a factor of 2 .

Circuit Complexity vs. Number of ANDs in Secure Computation. As explained above the circuit complexity of a function $f:\{0,1\}^{n} \times\{0,1\}^{n} \rightarrow\{0,1\}$ provides an upper bound on the number of ANDs required for secure computation of $f$ by a randomized protocol. It might seem tempting to think that the circuit complexity characterizes the number of ANDs. However, this is not true. 
There are functions with high circuit complexity which require few or no ANDs. For example, $f$ can be a function only of Alice's input with high circuit complexity which Alice can compute securely without any communication or calls to the AND black box. Furthermore, our results show that circuit complexity does not characterizes the number of ANDs required to securely compute a function by a deterministic protocol (this number of ANDs can be larger or smaller than the circuit complexity).

\section{Preliminaries}

In this section we define one-sided information-theoretic secure two-party computation in the honest-but-curios model. In our definition we allow the parties to execute a black box to a pre-defined function.

Protocols. We consider a two-party protocol with a pair of parties (Turing Machines), Alice and Bob. They have an access to a black box BB which computes some function $\mathrm{BB}: D_{1} \times D_{2} \rightarrow D_{3}$. Briefly, on inputs $(x, y)$, where $x$ is a private input for Alice and $y$ a private input for Bob, and random inputs $\left(r_{A}, r_{B}\right)$, where $r_{A}$ is a private random tape for Alice and $r_{B}$ is a private random tape for Bob, protocol (Alice, Bob) computes its output in a sequence of rounds of three types: Alice's rounds, Bob's rounds, and black-box rounds. In an Alice's round (respectively, Bob's round) only Alice (respectively, only Bob) is active and sends a message (i.e., a string) that will become an available input to Bob (respectively, to Alice) in the next round. In a black-box round Alice puts a value $a \in D_{1}$ to a register and Bob puts a value $b \in D_{2}$ to a register. In the end of this round Alice gets the value $\mathrm{BB}(a, b)$ in a third register, and Bob gets no information. A computation of Alice and Bob ends in a round in which Alice computes a private output. In this paper we focus on an AND black box, where AND: $\{0,1\} \times\{0,1\} \rightarrow\{0,1\}$ and $\operatorname{AND}(a, b)=a \wedge b$.

Transcripts, Views, and Outputs. Letting $E$ be an execution of protocol (Alice, Bob) on inputs $(x, y)$ and random inputs $\left(r_{A}, r_{B}\right)$, we make the following definitions:

- The transcript of $E$ consists of the sequence of messages exchanged by Alice and Bob, and is denoted by $\operatorname{TRANS}\left(x, r_{A}, y, r_{B}\right)$;

- The black-box outputs of $E$ consists of the outputs of the black box during the execution of the protocol, and is denoted by $\operatorname{BLACK}-\operatorname{BOX}\left(x, r_{A}, y, r_{B}\right)$;

- The view of Alice consists of the quadruplet

$$
\left(x, r_{A}, \operatorname{TRANS}\left(x, r_{A}, y, r_{B}\right), \operatorname{BLACK}-\operatorname{BOX}\left(x, r_{A}, y, r_{B}\right)\right),
$$

and is denoted by $\operatorname{VIEW}_{\text {Alice }}\left(x, r_{A}, y, r_{B}\right)$;

- The view of Bob consists of $\left(y, r_{B}\right.$, TRANS $\left.\left(x, r_{A}, y, r_{B}\right)\right)$, and is denoted by $\operatorname{VIEW}_{\mathrm{Bob}}\left(x, r_{A}, y, r_{B}\right)$.

We consider the random variables $\operatorname{TRANS}\left(x, \cdot, y, r_{B}\right), \operatorname{TRANS}\left(x, r_{A}, y, \cdot\right)$, and $\operatorname{TRANS}(x, \cdot, y, \cdot)$, respectively obtained by randomly selecting $r_{A}, r_{B}$, or 
both, and then outputting $\operatorname{TRANS}\left(x, r_{A}, y, r_{B}\right)$. We also consider the similarly defined random variables for VIEW $\mathrm{Alice}_{\text {and }}$ VIEW $\mathrm{Bob}_{\mathrm{B}}$.

In the model we consider, the two-party honest-but-curious model, each party is curious, that is, it may try to deduce as much information possible from its own view of an execution about the other's private input. However, each party is honest, that is, it scrupulously follows the instructions of the protocol. In such conditions, it is easy to enforce the correctness condition (for securely computing a function $f$ ), but not necessarily the privacy conditions. Note that, unlike secure computation in the malicious model, in the honest-but-curious model we can separate the security requirement into two separate requirements: correctness and privacy.

In the following definition we consider partial functions $f: A \times B \rightarrow C \cup\{*\}$, where $A, B$ and $C$ are some finite sets and $* \notin C$. If $f(x, y)=*$ then we say that $f$ is undefined on $x, y$. The reason that we consider partial functions is that in Section 3 we use them to characterize the number of ANDs required to securely compute fully-defined functions. To define the privacy in a protocol we consider the statistical distance between two distributions $Y_{0}, Y_{1}$ which is defined by $\operatorname{DIST}\left(Y_{0}, Y_{1}\right)=\frac{1}{2} \sum_{y}\left|\operatorname{Pr}\left[Y_{0}=y\right]-\operatorname{Pr}\left[Y_{1}=y\right]\right|$.

Definition 1 (Secure Computation). Let $f: A \times B \rightarrow C \cup\{*\}$ be a function, and $0 \leq \epsilon, \delta \leq 1$. A protocol (Alice, Bob) $(\epsilon, \delta)$-securely computes $f$, if the following conditions hold:

Correctness. For every $x \in A$ and every $y \in B$, if $f(x, y) \neq *$, then the probability that the output of Alice with $\operatorname{VIEW}_{\text {Alice }}(x, \cdot, y, \cdot)$ is $f(x, y)$ is at least $1-\epsilon$, where the probability is taken over $r_{A}$ and $r_{B}$.

Bob's Privacy. $\forall x \in A, \forall y_{0}, y_{1} \in B, \forall r_{A}$, if $f\left(x, y_{0}\right)=f\left(x, y_{1}\right) \neq *$ then

$$
\operatorname{DIST}\left(\operatorname{VIEW} \text { Alice }\left(x, r_{A}, y_{0}, \cdot\right), \operatorname{VIEW}_{\text {Alice }}\left(x, r_{A}, y_{1}, \cdot\right)\right) \leq \delta .
$$

Alice's Privacy. $\forall x_{0}, x_{1} \in A, \forall y \in B, \forall r_{B}$, if $f\left(x_{0}, y\right) \neq *$ and $f\left(x_{1}, y\right) \neq *$, then

$$
\operatorname{DIST}\left(\operatorname{VIEW} \mathrm{Bob}_{\mathrm{Bob}}\left(x_{0}, \cdot, y, r_{B}\right), \operatorname{VIEW}_{\mathrm{Bob}}\left(x_{1}, \cdot, y, r_{B}\right)\right) \leq \delta .
$$

A protocol securely computes $f$ if it $(0,0)$-securely computes $f$. In this case, we also say that the protocol computes $f$ with perfect security. A protocol is deterministic if Alice's and Bob's moves in the protocol do not depend on their random inputs.

Notice that the requirements in Alice's privacy and in Bob's privacy are not symmetric. We require that Alice's privacy is protected for all inputs where $f$ is defined. As Alice learns the output of $f$, we require that Bob's privacy is protected only when $f\left(x, y_{0}\right)=f\left(x, y_{1}\right) \neq *$.

The main measure we consider is the number of calls to the black box during a protocol.

Definition 2 (Number of ANDs). The number of calls to the AND black box in a protocol is the maximum over the inputs $x$ and $y$ and random inputs $r_{A}$ and $r_{B}$ of the number of black-box rounds in the execution with $x, y, r_{A}$, and $r_{B}$. 
Beimel, Micali, and Malkin [3], following Kushilevitz [22], characterize which functions can be computed securely without any calls to the AND black box. Their characterization uses the following notation and definitions. We represent a function $f: A \times B \rightarrow C \cup\{*\}$ by a matrix $M_{f}$ whose rows are labeled by the elements of $A$, columns are labeled by the elements of $B$, and $M_{f}(x, y)=f(x, y)$.

Definition 3 (Insecure Minor). A matrix contains an insecure minor if there are $x_{0}, x_{1}, y_{0}, y_{1}$ such that $M\left(x_{0}, y_{0}\right)=M\left(x_{0}, y_{1}\right) \neq *, M\left(x_{1}, y_{0}\right), M\left(x_{0}, y_{1}\right) \neq *$, and $M\left(x_{1}, y_{0}\right) \neq M\left(x_{0}, y_{1}\right)$.

The following theorem of [3] states that a function can be computed securely without ANDs iff it does not contain an insecure minor.

Theorem 1 ([3]). The function $f$ can be computed by a perfectly-secure randomized protocol with 0 ANDs if and only if the function $f$ can be computed by a deterministic protocol with 0 ANDs if and only if $M_{f}$ does not contain an insecure-minor.

The next definition is helpful for characterizing the number of required ANDs, by defining a relation on the columns of the matrix $M_{f}$.

Definition 4 ([2] $)$. The relation $\sim_{C}$ on the columns of a matrix $M$ is defined as follows: $y, y^{\prime} \in B$ satisfy $y \sim_{C} y^{\prime}$ if there exists some $x \in A$ such that $M(x, y)=M\left(x, y^{\prime}\right) \neq *$. The equivalence relation $\equiv_{C}$ on the columns of $M$ is defined as the transitive closure of the relation $\sim_{C}$. That is, $y \equiv_{C} y^{\prime}$, for $y, y^{\prime} \in B$, if there are $y_{1}, \ldots$, y $y_{\ell}$ such that $y \sim_{C} y_{1} \sim_{C} y_{2} \sim_{C} \ldots \sim_{C} y_{\ell} \sim_{C} y^{\prime}$.

In the rest of this section we prove various properties of secure protocols used throughout the paper. We next relate the number of ANDs required to securely compute a function, to the number of ANDs required to securely compute the functions restricted to each equivalence class. The proof of the following lemma appears in the full version of the paper.

Lemma 1. Let $f: A \times B \rightarrow C \cup\{*\}$ be a function, let $B_{1}, \ldots, B_{k}$ the equivalence classes of the relation $\equiv_{C}$, and define $f_{i}: A \times B_{i} \rightarrow C \cup\{*\}$ as the restriction of $f$ to $B_{i}$ The function $f$ can be computed securely by a randomized protocol (respectively, deterministic protocol) with $q$ ANDs if and only if each function $f_{i}$ can be computed securely by a randomized protocol (respectively, deterministic protocol) with $q$ ANDs.

For the results in this paper, we need the following standard result. Informally, the lemma asserts that if the columns of $M_{f}$ are equivalent then in perfectly-secure protocols no information is disclosed by the communication, and all the information that Alice needs to compute the function is passed through the outputs of the black box alone.

Lemma 2. Let $f: A \times B \rightarrow C$ be a function s.t. all columns of $M_{f}$ are equivalent and let $c$ be any communication transcript that can be exchanged between Alice and Bob in a protocol with perfect privacy. Then for every $x, x^{\prime} \in A$ and every $y, y^{\prime} \in B$ it holds that $\operatorname{Pr}[c=\operatorname{TRANS}(x, \cdot, y, \cdot)]=\operatorname{Pr}\left[c=\operatorname{TRANS}\left(x^{\prime}, \cdot, y^{\prime}, \cdot\right)\right]$, where the probability is taken over the random inputs of Alice and Bob. 
The proof of Lemma 2 is omitted. Recall that in any deterministic protocol, for every $x, y$ there is one possible communication transcript. Thus, by Lemma 2 if all the columns of $M_{f}$ are equivalent, then the same transcript will be exchanged for every pair of inputs. Thus, in deterministic protocols Alice and Bob can discard the communication and only execute the AND black boxes.

Lemma 3. Let $f: A \times B \rightarrow C$ be a function s.t. all columns of $M_{f}$ are equivalentIn every deterministic secure protocol there is exactly one communication transcript that is exchanged between Alice and Bob for all inputs $x, y$.

\section{Deterministic Protocols}

In this section we examine how many ANDs are needed to compute a function securely by a deterministic protocol. We start by giving an exact characterization of the functions that can be securely computed by deterministic protocols with $q$ ANDs. This characterization proves that there is a complete hierarchy of functions according to the number of ANDs. In particular, we establish that every function can be computed securely by a deterministic protocol provided that enough ANDs are executed. This should be contrasted to the malicious model where it is known that randomization is required [14].

For finite functions one can find the optimal protocol using our characterization. However, in general, our characterization does not lead to an efficient algorithm that determines how many ANDs are required to compute a function securely. Therefore, in Theorem 3 we give a simple and explicit upper bound on the number of ANDs that are required. Finally, we show in Theorem 4 that this upper bound is tight for Boolean functions. We note that our upper bound seems to be impractical since the number of ANDs can be exponential in the length of the input. However, at least for Boolean functions, our lower bound proves that this is unavoidable if we consider deterministic protocols.

To characterize what can be done with $q$ ANDs by a deterministic protocol, we note that first Alice and Bob call the AND black box once, and then execute a protocol with $q-1$ ANDs to compute a related function described in Figure[1] For the first execution there are sets $A_{1} \subseteq A$ and $B_{1} \subseteq B$ such that Alice gets output one from the AND black box if and only if $x, y \in A_{1} \times B_{1}$. We have two requirements: (1) Alice does not learn any extra information from the output of the first AND black box, and (2) Alice and Bob can compute the following function $f_{A_{1}, B_{1}}$ using $q-1$ ANDs. Formally, given a function $f$ : $A \times B \rightarrow C \cup\{*\}$ and two sets $A_{1} \subseteq A$ and $B_{1} \subseteq B$ we define a function $f_{A_{1}, B_{1}}:\left(A \cup\left(A_{1} \times\{1\}\right)\right) \times B \rightarrow C \cup\{*\}$, described in Figure 1, as follows:

1. $f_{A_{1}, B_{1}}(x, y)=f(x, y)$ for every $x \in A \backslash A_{1}$ and every $y \in B$.

2. $f_{A_{1}, B_{1}}(x, y)=f(x, y)$ for every $x \in A_{1}$ and every $y \in B \backslash B_{1}$.

3. $f_{A_{1}, B_{1}}(x, y)=*$ for every $x \in A_{1}$ and every $y \in B_{1}$.

4. $f_{A_{1}, B_{1}}(\langle x, 1\rangle, y)=*$ for every $x \in A_{1}$ and every $y \in B \backslash B_{1}$.

5. $f_{A_{1}, B_{1}}(\langle x, 1\rangle, y)=f(x, y)$ for every $x \in A_{1}$ and every $y \in B_{1}$. 

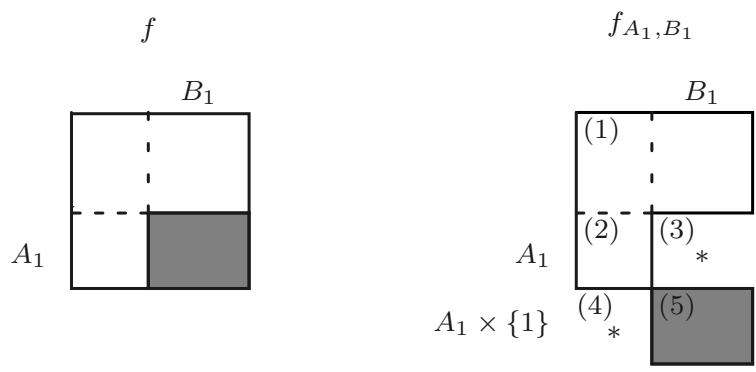

Fig. 1. The matrices of the functions $f$ and $f_{A_{1}, B_{1}}$. The numbers in the description of $f_{A_{1}, B_{1}}$ refer to the different cases in its definition.

Theorem 2. Let $f: A \times B \rightarrow C \cup\{*\}$ be a function such that all columns of $M_{f}$ are equivalent according to $\equiv_{C}$. The function $f$ can be computed securely with $q$ calls to the $A N D$ black box if and only if there are sets $A_{1} \subseteq A$ and $B_{1} \subseteq B$ such that the following two requirements hold:

1. For every $x \in A_{1}$, every $y_{0} \notin B_{1}$, and every $y_{1} \in B_{1}$ such that $f\left(x, y_{0}\right), f\left(x, y_{1}\right) \neq *$ it holds that $f\left(x, y_{0}\right) \neq f\left(x, y_{1}\right)$, and

2. The function $f_{A_{1}, B_{1}}$ can be computed securely with $q-1$ calls to the AND black box.

Proof. We first prove that the above conditions are sufficient. Assume the conditions hold. The secure protocol for computing $f$ proceeds as follows:

- Alice and Bob call the AND black box where Alice puts 1 iff $x \in A_{1}$ and Bob put 1 iff $y \in B_{1}$.

- Alice and Bob execute the secure protocol for $f_{A_{1}, B_{1}}$ with $q-1$ calls to the AND black box, where Bob's input is $y$ and Alice's input is $\langle x, 1\rangle$ if the AND output is 1 and $x$ otherwise.

- Alice's output is the output of the protocol for $f_{A_{1}, B_{1}}$.

We first argue that the protocol is correct. On one hand, if the output of the AND black box is 1 , then $x \in A_{1}$ and $y \in B_{1}$. Thus, by the definition of $f_{A_{1}, B_{1}}$ it holds that $f_{A_{1}, B_{1}}(\langle x, 1\rangle, y)=f(x, y)$, and the output of the protocol is correct. On the other hand, if the output of the AND black box is 0, then either $x \notin A_{1}$ or $y \notin B_{1}$. Thus, $f_{A_{1}, B_{1}}(x, y)=f(x, y)$, and the output of the protocol is correct. Note that the protocol never tries to evaluate $f_{A_{1}, B_{1}}$ on inputs where it is not defined.

To argue that the protocol is perfectly-secure, first note that Bob gets no messages during the first step of the protocol, and he does not get any information from the black box. This guarantees Alice's Privacy. To argue about Bob's privacy, note that Alice learns information about $y$ from the first call to the AND black box only if $x \in A_{1}$. In this case, by Condition 1. Alice learns if $y \in B_{1}$ from the output of the function $f$ itself. Thus, this step is secure, and Alice is allowed to know the output of the black box and the output of $f_{A_{1}, B_{1}}$ which as 
argued is equal to the desired output of $f$. Finally, as the protocol for $f_{A_{1}, B_{1}}$ is secure, the entire protocol for $f$ is secure.

We next prove that the conditions of the theorem are necessary. Assume that $f$ can be computed securely with $q$ ANDs. By Lemma 3, we can assume w.l.o.g. that Alice and Bob do not exchange any messages, and all information Alice gets is through the outputs of the calls to the AND black boxes. Let $A_{1}$ and $B_{1}$ be the sets of inputs of Alice and Bob respectively for which they put 1 to the first call to the AND black box. Condition 1 must hold or otherwise Alice learns extra information from the answer of the first AND. As for Condition 2, we can use the following protocol to compute $f_{A_{1}, B_{1}}$ securely with $q-1$ ANDs: Alice and Bob execute the protocol for $f$ with the following two changes: (1) If Alice's "real" input is $\langle x, 1\rangle$ for $x \in A_{1}$ then she replaces it by the input $x$, and (2) the first call to the AND black box is not executed. Instead, Alice simulates it by considering its output as 1 if her input is $\langle x, 1\rangle$ and 0 otherwise. The rest of the protocol is executed without any changes. As the protocol for $f$ uses $q$ ANDs, and Alice and Bob do not use the first AND, the resulting protocol for $f_{A_{1}, B_{1}}$ uses $q-1$ ANDs as required.

Our next theorem gives a simple upper bound on the number of ANDs required to compute a function securely. The proof of this upper-bound gives a simple secure protocol for computing the function.

Theorem 3. Let $f: A \times B \rightarrow C \cup\{*\}$ be a function. The function $f$ can be computed securely by a deterministic protocol with $|A|\lceil\log |C|\rceil A N D s$.

Proof. First assume that $f$ is Boolean, i.e., $C=\{0,1\}$. We next describe a protocol which uses $|A|$ ANDs. Assume the input of Alice is $x$ and the input of Bob is $y$. For every $z \in A$, Alice and Bob execute the AND black box, where Alice puts 1 to the AND if $x=z$ and 0 otherwise, and Bob puts $f(z, y)$ to the AND. Alice outputs the output of the AND corresponding to $x$, that is, $\operatorname{AND}(1, f(x, y))=f(x, y)$ as required. Bob does not gain any information during this protocol (since there is no communication and only Alice gets the output of the black box) and Alice only gains $f(x, y)$.

If $|C|>2$, then we consider the binary representation of $f(x, y)$ (of length exactly $\lceil\log |C|\rceil)$, and execute the above protocol for every bit of $f(x, y)$.

The following theorem shows that the upper bound of Theorem 3 is tight for every Boolean function. In the theorem we assume that there is some $y_{0}$ such that $f\left(x, y_{0}\right)=0$ for every $x \in A$. This assumption is without loss of generality since Alice learns the output of the protocol and knows $x$, thus she can use any renaming of the outputs in every row.

Theorem 4. Let $f: A \times B \rightarrow\{0,1\}$ be a Boolean function such that all the rows of $M_{f}$ are distinct and non-constant, there is some $y_{0} \in B$ such that $f\left(x, y_{0}\right)=0$ for every $x \in A$, and all of its columns are equivalent according to $\equiv_{C}$. Then, every deterministic protocol computing $f$ securely must use at least $|A| A N D s$.

Proof. Fix any deterministic protocol that computes $f$ securely. By Lemma 3, we can assume, without loss of generality, that Alice and Bob do not exchange any 
messages and the view of Alice includes her input and the outputs of the black box. Consider any $x \in A$. Since $f$ is Boolean there are exactly two views Alice should see given $x$ : one view for every $y$ such that $f(x, y)=0$ and another view for every $y$ such that $f(x, y)=1$. For every $x$, consider the first black-box call where Alice can get two different answers. As argued above one output corresponds to the case where $f(x, y)=0$ and the other output corresponds to the case where $f(x, y)=1$. Thus, Alice can deduce the output of the function $f(x, y)$ from this black-box answer and, therefore, we say that this is the significant call to the AND black box for $x$.

Assume, towards contradiction, that for two different $x_{0}, x_{1} \in A$ the significant call is the same. Recall that $f\left(x_{0}, y_{0}\right)=f\left(x_{1}, y_{0}\right)=0$, and since the rows corresponding to $x_{0}$ and $x_{1}$ are not the same, there is some $y_{1}$ such that, w.l.o.g., $f\left(x_{0}, y_{1}\right)=0$ while $f\left(x_{1}, y_{1}\right)=1$. Bob has to put the same value to this significant call when he holds $y_{0}$ and $y_{1}$ or Alice would learn information when she holds $x_{0}$. This means that Alice cannot compute the correct value of $f\left(x_{1}, y_{0}\right)$ or $f\left(x_{1}, y_{1}\right)$ since in both cases she gets the same information, contradiction.

To conclude, for every $x \in A$ there is a unique significant call to the AND black box, thus, there are at least $|A|$ calls to the AND black box.

In the protocol implied by Theorem 3 Alice is non-adaptive as her inputs to the AND black box depend only on her input and not on the outputs of previous AND black boxes. In Theorem 4 we prove that for Boolean functions this is optimal. However, the protocol implied by Theorem 2 is adaptive, and for non-Boolean functions adaptively does help (namely the bound is not tight), as shown in the following example. Consider the function $f:\{0,1,2\} \times\{0,1,2,3\} \rightarrow$ $\{0,1,2\}$ described in Figure 2 We next describe a secure protocol for $f$ which

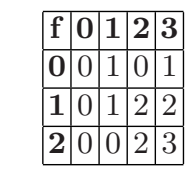

The function $f$

\begin{tabular}{|c|c|c|c|c|}
\hline $\mathbf{f}_{\mathbf{A}_{\mathbf{1}}, \mathbf{B}_{\mathbf{1}}}$ & $\mathbf{0}$ & $\mathbf{1}$ & $\mathbf{2}$ & $\mathbf{3}$ \\
\hline $\mathbf{0}$ & 0 & 1 & 0 & 1 \\
\hline $\mathbf{1}$ & 0 & 1 & $*$ & $*$ \\
\hline $\mathbf{2}$ & 0 & 0 & $*$ & $*$ \\
\hline$\langle\mathbf{1}, \mathbf{1}\rangle$ & $*$ & $*$ & 2 & 2 \\
\hline$\langle\mathbf{2}, \mathbf{1}\rangle$ & $*$ & $*$ & 2 & 3 \\
\hline
\end{tabular}

The function $f_{A_{1}, B_{1}}$

Fig. 2. The functions $f$ and $f_{A_{1}, B_{1}}$.

uses two ANDs. For the first AND, Alice puts 1 if $x \in A_{1}=\{1,2\}$ and Bob puts 1 if $y \in B_{1}=\{2,3\}$. After this AND Alice and Bob need to securely compute the function $f_{A_{1}, B_{1}}$ described in Figure 2. Computing $f_{A_{1}, B_{1}}$ is done using a second AND where Alice puts 1 if $x \in A_{2}=\{0,1,\langle 2,1\rangle\}$ and Bob puts 1 if $y \in B_{2}=\{1,3\}$. After this AND, Alice can deduce the output of $f$ from her input and the outputs of the ANDs. In this protocol Alice is adaptive; with input 1 , for example, she puts 1 to the second AND if the output of the first AND was 0 and she puts 0 otherwise. 


\section{Randomized Protocols}

In this section we investigate the power of randomization in our setting. We show that, in general, randomization helps: the gap between the number of ANDs required by a randomized protocol and a deterministic one may be exponential. We also quantify how much randomization can help, and study its limits. Finally, we show that allowing a statistically secure protocol with some error probability may significantly reduce the number of ANDs compared to the number required by a perfect randomized protocol.

\subsection{Randomization Helps}

The following theorem, adapted from [16, establishes an upper bound on the number of ANDs needed to securely compute a function, in terms of the number of gates in its circuit. Together with our characterization for deterministic protocols in the previous section, the theorem proves that randomization helps, as we elaborate below.

Theorem 5 ([16]). If $f$ can be computed by a Boolean circuit with fan-in 2 whose size is $s$, then there is a perfectly-secure randomized protocol computing $f$ which uses $4 s$ AND calls.

Proof. Theorem 5] is proved in [16] by having each of the parties additively secretshare their inputs, and then processing the shares through each of the gates in the circuit. Depending on the gate, the parties may need to use the primitive of 1-out-of-4 Oblivious Transfer, which can be implemented using four ANDs.

We next describe the protocol in our context. Alice and Bob compute the function $f$ one gate at a time, such that for each wire in the circuit, Alice and Bob hold two random bits whose exclusive-or is the correct value for that wire in a non-secure computation of the circuit (see Figure [3). For initialization, for every variable $x_{i}$ held by Alice, the bits held by Alice and Bob respectively are $\left(s_{A}, s_{B}\right)$ where Alice holds the bit $s_{A}=x_{i}$ and Bob holds the bit $s_{B}=0$. The variables held by Bob are dealt symmetrically. We next explain how to compute a Boolean gate $G$ where the correct values of its inputs computed by the circuit are $s 1$ and $s 2$ and the correct value of the output of the gate is $s 3=G(s 1, s 2)$. Before the computation of the gate Alice holds $\left(s 1_{A}, s 2_{A}\right)$ and Bob holds $\left(s 1_{B}, s 2_{B}\right)$ such that $s 1=s 1_{A} \oplus s 1_{B}$ and $s 2=s 2_{A} \oplus s 2_{B}$. At the end of the computation Alice and Bob should hold random bits $\left(s 3_{A}, s 3_{B}\right)$ such that $s 3=s 3_{A} \oplus s 3_{B}$. To compute the gate, Bob chooses a random bit $s 3_{B}$, and computes the value of $s 3_{A}$ for the 4 possible values $(0,0),(0,1),(1,0)$, and $(1,1)$ of Alice's inputs $\left(s 1_{A}, s 2_{A}\right)$. That is, Bob computes for every $a 1, a 2 \in\{0,1\}$ the value $s 3_{A}=s 3_{B} \oplus G\left(a 1 \oplus s 1_{B}, a 2 \oplus s 2_{B}\right)$. Thereafter, Alice and Bob perform four ANDs, corresponding to the possible values of Alice's inputs, where Alice puts 1 to the AND execution corresponding to her true inputs $\left(s 1_{A}, s 2_{A}\right)$, and 0 to the other three, and Bob puts the values of $s 3_{A}$ he computed. For the final gate application, Bob chooses $s 3_{B}=0$, so that Alice's output for that gate (in the appropriate AND execution) is the output of the function. The correctness and privacy of this protocol are easy to verify. 


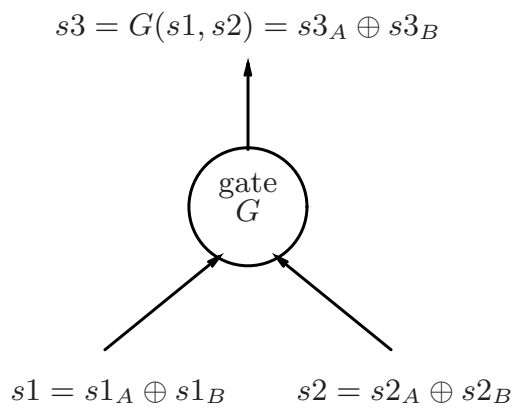

Fig. 3. A secure evaluation of a gate $G$.

The above theorem applies for circuits with gates which are arbitrary Boolean functions with fan-in 2. Depending on the circuit, the theorem can be optimized to achieve a smaller number of ANDs, as some of the gates may require only 2 ANDs (when one of the incoming wires is from Bob's initial inputs) or no ANDs (when the gate computes exclusive-or). Such optimizations are used in the following examples to obtain slightly better parameters than guaranteed by a direct application of the theorem as stated.

We conclude that randomization helps for functions where the upper bound promised by Theorem 5 for randomized protocols is smaller than the lower bound established in Theorem 2 for deterministic protocols. We next provide a few concrete examples, which exhibit when and how much randomization helps.

Example 1 (Inner Product $\mathrm{IP}_{\mathbf{n}}$ ). Let $\operatorname{IP}_{n}:\{0,1\}^{n} \times\{0,1\}^{n} \rightarrow\{0,1\}$ be the inner-product modulo 2 function, that is, $\operatorname{IP}_{n}(x, y)=\oplus_{i=1}^{n} x_{i} y_{i}$. We show that the function $\mathrm{IP}_{n}$ can be computed with $2 n$ ANDs using a perfect randomized protocol, but requires at least $2^{n}-1$ ANDs in any deterministic protocol.

Lemma 4. The function $\mathrm{IP}_{n}$ can be securely computed with $2 n$ ANDs using a randomized protocol. Any deterministic protocol for securely computing $\mathrm{IP}_{n}$ requires at least $2^{n}-1$ ANDs (and there is a deterministic protocol using this number of $A N D s)$.

Proof. Consider the following protocol on input $x=x_{1}, \ldots, x_{n}$ for Alice and $y=y_{1}, \ldots, y_{n}$ for Bob. Bob chooses $r_{1}, \ldots, r_{n-1} \in\{0,1\}^{n}$ uniformly at random and sets $r_{n} \leftarrow \oplus_{i=1}^{n-1} r_{i}$. Then, for each $i=1, \ldots, n$, Alice and Bob run two ANDs, as follows: $a_{i}^{0} \leftarrow \wedge\left(1-x_{i}, r_{i}\right)$ and $a_{i}^{1} \leftarrow \wedge\left(x_{i}, y_{i} \oplus r_{i}\right)$. Alice outputs $\oplus_{i=1}^{n} a_{i}^{x_{i}}=\operatorname{IP}_{n}(x, y)$. The claims about deterministic protocols for $\operatorname{IP}_{n}$ follow from Theorem 4 and Theorem 3 .

As we will explain in Example 5, the number of ANDs in this protocol is tight up to a constant, since every randomized protocol for $\mathrm{IP}_{n}$ requires at least $n / 2$ ANDs even if we allow errors and statistical privacy. We next show a tradeoff between the number of random bits and the number of ANDs. 


\section{A randomized protocol for $\mathrm{IP}_{3 \downarrow} \downarrow \frac{}{(1,1,1)}$ with 4 ANDs}

Alice's input: $x_{1}, x_{2}, x_{3}$ where the number of variables with value 1 is $\leq 2$.

Bob's input: $y_{1}, y_{2}, y_{3}$

Alice's desired output: $x_{1} y_{1} \oplus x_{2} y_{2} \oplus x_{3} y_{3}$

Bob chooses $r$ at random from $\{0,1\}$.

Alice sets $a=1$ iff exactly one of her inputs has value 1 .

Alice and Bob execute the following 4 ANDs:

$$
\begin{array}{ll}
a_{1} \leftarrow \wedge\left(x_{1}, y_{1} \oplus r\right), & a_{2} \leftarrow \wedge\left(x_{2}, y_{2} \oplus r\right), \\
a_{3} \leftarrow \wedge\left(x_{3}, y_{3} \oplus r\right), & a_{4} \leftarrow \wedge(a, r) .
\end{array}
$$

Alice's ouput: $a_{1} \oplus a_{2} \oplus a_{3} \oplus a_{4}$.

Fig. 4. A randomized protocol with 4 ANDs for a function requiring 6 ANDs in any deterministic protocol.

Lemma 5. The function $\mathrm{IP}_{n}$ can be securely computed using $R-1$ random bits and $R 2^{\lceil n / R\rceil} A N D$ s, for all $1 \leq R \leq n$.

Proof. The protocol is a generalization of the protocol described in the proof of Lemma 4. Denote $n^{\prime}=\lceil n / R\rceil$. Bob chooses $R-1$ random bits $r_{1}, \ldots, r_{R-1}$ and sets $r_{R} \leftarrow \oplus_{i=1}^{R-1} r_{i}$. Then, for $i=0$ to $R-1$ Alice and Bob compute the function $a_{i} \leftarrow \operatorname{IP}\left(\left\langle x_{i n^{\prime}+1}, \ldots, x_{(i+1) n^{\prime}}\right\rangle,\left\langle y_{i n^{\prime}+1}, \ldots, y_{(i+1) n^{\prime}}\right\rangle\right) \oplus r_{i}$ using the secure deterministic protocol of Theorem 3 , which uses $2^{\lceil n / R\rceil}$ ANDs, where Alice's input is $\left\langle x_{i n^{\prime}+1}, \ldots, x_{(i+1) n^{\prime}}\right\rangle$ and Bob's input is $\left\langle y_{i n^{\prime}+1}, \ldots, y_{(i+1) n^{\prime}}\right\rangle, r_{i}$. Alice outputs the value $\oplus_{i=0}^{R-1} a_{i}$ which by the properties of IP and the choice of the $r_{i}$ 's is the correct value. Since the first $R-1$ random bits are chosen independently and the deterministic IP protocol is secure, the protocol we construct is secure.

Example 2 (Restricted $\mathrm{IP}_{\mathbf{3}}$ ). Consider the restricted-domain inner product function $\mathrm{IP}_{3}$, where Alice's input cannot be $x=(1,1,1)$, and denote it by $\operatorname{IP}_{3} \downarrow \overline{(1,1,1)}$. We show in Figure 4 that this function can be computed with 4 ANDs in a randomized protocol with perfect privacy and correctness, but requires 6 ANDs in any deterministic protocol. We note that 4 is the smallest number of ANDs for which we can prove that randomization helps (in Section 4.3 we will see that for one AND we can prove randomization does not help). We leave as an open problem whether randomization helps or not for the case of 2 or 3 ANDs.

Lemma 6. The function $\mathrm{IP}_{3} \downarrow \frac{}{(1,1,1)}$ can be securely computed with 4 ANDs in a randomized protocol, but the minimal number of ANDs required by a deterministic protocol for this function is 6 .

Example 3 (Equality $\mathrm{EQ}_{n}$ ). Let $\mathrm{EQ}_{n}:\{0,1\}^{n} \times\{0,1\}^{n} \rightarrow\{0,1\}$ be the equality function, that is, $\operatorname{EQ}_{n}(x, y)=1$ iff $x=y$. We show below that the number of ANDs required to compute the function $\mathrm{EQ}_{n}$ using a perfect deterministic protocol is exponential in $n$, while using a perfect randomized protocol this number 


\section{A randomized (imperfect) protocol with for $\mathrm{EQ}_{n}$}

Alice's input: $x=x_{1}, \ldots, x_{n} \in\{0,1\}^{n}$

Bob's input: $y=y_{1}, \ldots, y_{n} \in\{0,1\}^{n}$

Alice's desired output: $\mathrm{EQ}_{n}(x, y)$

Bob chooses $k$ vectors $\boldsymbol{r}^{1}, \ldots, \boldsymbol{r}^{k} \in\{0,1\}^{n}$ uniformly at random,

Bob sends $\boldsymbol{r}^{1}, \ldots, \boldsymbol{r}^{k} \in\{0,1\}^{n}$ to Alice

Alice computes $a_{j}=\operatorname{IP}_{n}\left(x, \boldsymbol{r}^{j}\right)$ for $j=1, \ldots, k$, and sets $a=a_{1}, \ldots, a_{k}$

Bob computes $b_{j}=\operatorname{IP}_{n}\left(y, \boldsymbol{r}^{j}\right)$ for $j=1, \ldots, k$, and sets $b=b_{1}, \ldots, b_{k}$

Alice and Bob use the randomized prot. of Lemma 7 to compute $\mathrm{EQ}_{k}(a, b)$.

Alice's ouput: the output of the protocol for $\operatorname{EQ}_{k}(a, b)$.

Fig. 5. A randomized protocol with $O(k)$ ANDs, $1 / 2^{k}$ error and $2 / 2^{k}$ distance for $\mathrm{EQ}_{n}$.

is linear in $n$, and using a randomized protocol with small error probability and statistical privacy, the number of ANDs is independent of $n$ and depends only on the allowed error and distance (which are exponentially small in the number of ANDs). The specific lemmas are stated below.

Lemma 7. Any deterministic protocol computing $\mathrm{EQ}_{n}$ must use at least $2^{n}$ ANDs, and there is a deterministic protocl with this number of ANDs. Any perfectly-secure randomized protocol computing $\mathrm{EQ}_{n}$ must use at least $n$ ANDs, and there exits such a protocol using $O(n)$ ANDs.

Proof. Noting that the matrix for $\mathrm{EQ}_{n}$ is the identity matrix, the upper bound for deterministic protocols follows directly from Theorem [3. The lower bound for deterministic protocols follows from Theorem 4 since the matrix satisfies all the conditions of the theorem (including the all-zero column, if we exchange the roles of 0 and 1 outputs in one of the rows). The upper bound for randomized protocols follows from Theorem [5] by noting that there is a Boolean circuit with fan-in 2 and with $O(n)$ gates that computes $\mathrm{EQ}_{n}$. The lower bound for randomized protocols follows from Theorem 6 below.

Lemma 8. For every $k$, the function $\mathrm{EQ}_{n}$ can be computed with $O(k)$ ANDs by a randimized protocol with $1 / 2^{k}$ error and at most $1 / 2^{k}$ statistical distance.

Proof. The protocl securely-computing $\mathrm{EQ}_{n}$ is described in Figure 5 The idea of the protocol is to approximately compare the initial $n$-bit inputs by (exactly) comparing $k$ inner products of the inputs with random strings, which as we saw can be done using $O(k)$ ANDs. It is clear that if $\mathrm{EQ}_{n}(x, y)=1$ (i.e., the inputs are equal) the protocol does not err. On the other hand, $\operatorname{Pr}\left[a^{j} \neq b^{j} \mid \mathrm{EQ}_{n}(x, y)=\right.$ $0]=1 / 2$ for every $j$, and since the vectors $r^{j}$ are chosen independently at random, $\operatorname{Pr}\left[\mathrm{EQ}_{k}(a, b)=1 \mid \mathrm{EQ}_{n}(x, y)=0\right]=1 / 2^{k}$, which establishes the error.

We next prove that the protocol has statistical privacy. Intuitively, Alice learns information only when she gets an incorrect output. Formally, fix any $x, y, y^{\prime} \in\{0,1\}^{n}$ such that $y \neq y^{\prime}$ and $\mathrm{EQ}_{n}(x, y)=\mathrm{EQ}_{n}\left(x, y^{\prime}\right)$, and compute 
the statistical distance between the view seen by Alice holding input $x$, when executing the protocol with Bob's input set to $y$ or to $y^{\prime}$ (we will denote the corresponding vectors computed in the protocol by $(a, b)$ and $\left(a^{\prime}, b^{\prime}\right)$ respectively). Observe that if $\mathrm{EQ}_{n}(x, y)=1, y$ and $y^{\prime}$ must be identical. Thus, we only need to consider the case where $\mathrm{EQ}_{n}(x, y)=0$, namely $x, y, y^{\prime}$ are three different vectors. The only information that Alice gets in the protocol which depends on Bob's input, is the output of the perfectly secure protocol for $\operatorname{EQ}_{k}(a, b)\left(\right.$ or $\left.\operatorname{EQ}_{k}\left(a^{\prime}, b^{\prime}\right)\right)$. This implies that given this output is the same, the views are distributed identically. On the other hand, we can bound the probability that this output is not the same, as follows.

$$
\begin{aligned}
& \operatorname{Pr}\left[\mathrm{EQ}_{k}(a, b) \neq \mathrm{EQ}_{k}\left(a^{\prime}, b^{\prime}\right) \mid \mathrm{EQ}_{n}(x, y)=0\right] \\
& \leq \operatorname{Pr}\left[\mathrm{EQ}_{k}(a, b)=1 \mid \mathrm{EQ}_{n}(x, y)=0\right]+\left[\mathrm{EQ}_{k}\left(a^{\prime}, b^{\prime}\right)=1 \mid \mathrm{EQ}_{n}(x, y)=0\right]=2 / 2^{k}
\end{aligned}
$$

We may therefore conclude that the statistical distance between Alice's views for input $(x, y)$ vs. $\left(x, y^{\prime}\right)$ is at most $1 / 2^{k}$. Finally, note that Bob does not receive any messages in this protocol, so Alice's perfect privacy follows immediately.

The number of ANDs used in the last lemma is independent of $n$, exhibiting an inherent gap between perfect and imperfect protocols. In order to get exponentially small statistical distance and error in this protocol, the number of ANDs should still be set to be linear in $n$, though it may be smaller than $n$. Setting the number of ANDs to be polylogarithmic in $n$ will already give a negligible statistical distance and error. This should be contrasted with the lower bounds of $n$ (or $2^{n}$ ) ANDs for perfect randomized (or deterministic, resp.) protocols for this function.

\subsection{How Much Does Randomization Help?}

In the previous section we showed that randomization can help significantly compared to deterministic protocols. In this section we consider the limitations of randomized protocols. We first show lower bounds on the number of ANDs required in randomized protocols. For a function $f:\{0,1\}^{n} \times\{0,1\}^{n} \rightarrow\{0,1\}^{n}$ our lower bound is at most $n$. Notice, that by Theorem 5 we cannot prove super-linear lower-bounds on the number of calls to the AND black box for explicit functions unless we prove super-linear lower-bounds for circuit complexity of explicit functions which is a long-standing open problem. We use our lower bounds to show that for Boolean functions the gap in the number of calls to the AND black box between deterministic protocols and randomized protocols with perfect security can be at most exponential.

We start by giving two lower bounds on the number of ANDs in perfectlysecure protocols. The proofs of these lower bounds is omitted for lack of space.

Theorem 6. Let $f: A \times B \rightarrow C$ be a function s.t. all columns of $M_{f}$ are equivalent and no two columns are the same. The number of AND black box calls in any perfectly-secure randomized protocol computing $f$ is at least $\lceil\log |B|\rceil$. 
Example $4\left(\left(\begin{array}{l}n \\ 1\end{array}\right) \mathrm{OT}\right)$. Consider the function $\left(\begin{array}{l}n \\ 1\end{array}\right) \mathrm{OT}:\{1, \ldots, n\} \times\{0,1\}^{n} \rightarrow\{0,1\}$ defined as $\left(\begin{array}{c}n \\ 1\end{array}\right) \mathrm{OT}\left(i,\left\langle y_{1}, \ldots, y_{n}\right\rangle\right)=y_{i}$. Theorem 6 proves that in any perfectlysecure protocol for $\left(\begin{array}{l}n \\ 1\end{array}\right)$ OT the number of ANDs is at least $n 3$ This implies that in any perfectly-secure protocol for $\left(\begin{array}{l}n \\ 1\end{array}\right)$ OT using an $\left(\begin{array}{l}2 \\ 1\end{array}\right)$ OT black box the number of $\left(\begin{array}{l}2 \\ 1\end{array}\right)$ OT is at least $n / 2$. This reproves the result of Dodis and Micali [14] up to a factor of 2 (our proof does not use information theory).

Theorem 7. Let $f: A \times B \rightarrow\{0,1\}$ be a Boolean function s.t. all columns of $M_{f}$ are equivalent, no two rows of $M_{f}$ are the same, and there is some $y_{0} \in B$ s.t. $f\left(x, y_{0}\right)=0$ for every $x \in A$. Then, the number of calls to the AND black box in any perfectly-secure randomized protocol computing $f$ is at least $\lceil\log |A|\rceil$.

The next theorem states that for Boolean functions the gap in the number of AND black-box calls between deterministic protocols and randomized protocols with perfect security is at most exponential. This seems to resemble the simple derandomization of randomized algorithms, however this resemblance is misleading (as executing a secure protocol with all possible random coins might leek information). As an example of the difficulty, the gap can be much larger for non-perfect randomized protocols. Another example is the malicious model where randomization is essential (see, e.g., 14]). We prove the gap between randomized and deterministic protocols by combining the lower bounds we proved on randomized protocols and the upper bounds for deterministic protocols.

Theorem 8. Let $f$ be a Boolean function. If there exists a perfectly-secure randomized protocol computing $f$ using $q$ ANDs then there is a deterministic protocol computing $f$ with $2^{q}$ ANDs.

Proof. By Lemma 1, the function $f$ can be securely computed with $q$ ANDs if and only if every equivalence class of the columns of $M_{f}$ can be computed securely with $q$ ANDs. Thus, by Theorem 7 , the number of distinct rows in any equivalence class is at most at most $2^{q}$. By Theorem 3 there is a deterministic protocol securely computing every equivalence class of $f$ using $2^{q}$ ANDs, and therefore, by Lemma 1 such protocol exists for $f$.

We next generalize Theorem [6 to protocols which might err with some probability. We first recall some definitions from communication complexity (for more information on this subject see [23]). The one-round randomized communication complexity in the public random coin model is defined as follows: Alice and Bob each have a private input and they have a shared random input. Bob sends one message to Alice, and Alice computes the output of the protocol (there are no privacy requirements). The error of the protocol is the probability that Alice outputs a value different than $f(x, y)$. A protocol computes $f$ with error $\epsilon$ if for every inputs $x, y$ its error is at most $\epsilon$. Let $R_{\epsilon}^{\text {pub, } B \rightarrow A}(f)$ be the number of communication bits in the best such protocol computing $f$ with error $\epsilon$.

${ }^{3}$ Using Theorem 9 below, one can prove that even in statistically-secure protocols for $\left(\begin{array}{l}n \\ 1\end{array}\right)$ OT the number of ANDs is $\Omega(n)$. 
Theorem 9. Let $f: A \times B \rightarrow\{0,1\}$ be a Boolean function s.t. all the columns of $M_{f}$ are equivalent and no two columns are identical. Then, in any randomized $(\epsilon, \delta)$-secure protocol computing $f$, the number of $A N D s$ is at least $R_{\epsilon+7 \delta}^{\mathrm{pub}, B \rightarrow A}(f)$.

The proof of Theorem 9 is omitted for lack of space. Theorem 6 is a special case of Theorem 9 since it easy to see that $R_{0}^{\text {pub, } B \rightarrow A}(f)=\lceil\log |B|\rceil$.

Example 5. By 15 it holds that $R_{\epsilon}^{\mathrm{pub}, B \rightarrow A}\left(\mathrm{IP}_{n}\right)=n / 2$ for every $\epsilon<1 / 2$. Thus, unlike $\mathrm{EQ}_{n}$, for every $\epsilon, \delta$ where $\epsilon+7 \delta<1 / 2$, the inner-product function does not have an $(\epsilon, \delta)$-secure protocol which uses less than $n / 2$ ANDs.

\subsection{One AND: Randomization Does Not Help}

We have seen that randomization can significantly reduce (up to an exponential factor) the number of required ANDs, and that already with 4 ANDs, randomized protocols compute a strictly stronger class of functions than deterministic protocols with the same number of ANDs. On the other hand, it is known (see Theorem (1) that for secure computation in our model without any ANDs, randomization does not help. In this section we show that with one AND randomization still does not help.

Theorem 10. Let $f: A \times B \rightarrow C$ be a function such that all the columns of $M_{f}$ are equivalent according to $\equiv_{C}$. The function $f$ can be computed securely by a randomized protocol using one call to the AND black box if and only if there are $A_{1} \subseteq A$ and $B_{1} \subseteq B$ such that:

1. For every $x \in A_{1}, y_{0} \notin B_{1}$, and $y_{1} \in B_{1}$ it holds that $f\left(x, y_{0}\right) \neq f\left(x, y_{1}\right)$,

2. For every $x \in A$ and every $y, y^{\prime} \in B$ such that either $x \notin A_{1}$ or $y, y \notin B_{1}$ it holds that $f(x, y)=f\left(x, y^{\prime}\right)$.

3. For every $x \in A_{1}$ and every $y, y^{\prime} \in B$ it holds that $f(x, y)=f\left(x, y^{\prime}\right)$.

Proof. First, if Conditions (11)-(3) hold then, by Theorem $2 f$ can be computed by a secure (deterministic) protocol with 1 AND. The function $f_{A_{1}, B_{1}}$ can be computed by Alice without any communication since each row of $f_{A_{1}, B_{1}}$ is constant.

For the other direction, assume there is a secure protocol that computes $f$ with 1 AND. Fix any communication string $c$ that has positive probability for some fixed inputs; by Lemma $2 c$ has positive probability given every $x, y$. Now, define $A_{1}=\{x: \operatorname{Pr}[$ Alice puts 1 to the black box with $x$ and communication $c]>0\}$, and $B_{1}=\{y: \operatorname{Pr}[$ Bob puts 1 to the black box with $y$ and communication $c]>0\}$. By the correctness and privacy requirements of the protocol $A_{1}$ and $B_{1}$ satisfy Conditions (11)-(3).

The protocol proving the sufficiency of the conditions in Theorem 10 is deterministic. Thus,

Corollary 1. Randomized protocols with one AND can compute securely exactly the same functions as deterministic protocols with one AND. 
Acknowledgments. We thank Yuval Ishai for helpful discussions and Enav Weinreb for helpful remarks on earlier versions of this paper. We are also grateful to AT\&T Labs-Research that hosted us for two weeks and for three years, respectively, during which part of this research was conducted.

\section{References}

1. D. Beaver. Perfect privacy for two-party protocols. Technical Report TR-11-89, Computer Science, Harvard University, 1989.

2. D. Beaver. Correlated pseudorandomness and the complexity of private computations. In the 28th Symp. on the Theory of Computing, pages 479-488, 1996.

3. A. Beimel, T. Malkin, and S. Micali. The all-or-nothing nature of two-party secure computation. In CRYPTO '99, volume 1666 of LNCS, pages 80-97. Springer, 1999.

4. M. Ben-Or, S. Goldwasser, and A. Wigderson. Completeness theorems for noncryptographic fault-tolerant distributed computations. In the 20th Symp. on the Theory of Computing, pages 1-10, 1988.

5. G. Brassard and C. Crépeau. Oblivious transfers and privacy amplification. In EUROCRYPT '97, volume 1233 of LNCS, pages 334-347. Springer, 1997.

6. G. Brassard, C. Crépeau, and J.-M. Robert. Information theoretic reductions among disclosure problems. In the 27th Symp. on Foundations of Computer Science, pages 168-173, 1986.

7. G. Brassard, C. Crépeau, and M. Sántha. Oblivious transfers and intersecting codes. IEEE Trans. on Information Theory, 42(6):1769-1780, 1996.

8. R. Canetti. Security and composition of multiparty cryptographic protocols. J. of Cryptology, 13(1):143-202, 2000.

9. D. Chaum, C. Crépeau, and I. Damgård. Multiparty unconditionally secure protocols. In the 20th Symp. on the Theory of Computing, pages 11-19, 1988.

10. B. Chor and E. Kushilevitz. A zero-one law for Boolean privacy. SIAM J. on Discrete Mathematics, 4(1):36-47, 1991.

11. C. Crépeau. Equivalence between two flavors of oblivious transfers. In CRYPTO '87, volume 293 of $L N C S$, pages 350-354. Springer, 1988.

12. C. Crépeau and J. Kilian. Achieving oblivious transfer using weakened security assumptions. In 29th Symp. on Found. of Computer Science, pp. 42-52, 1988.

13. I. Damgard, J. Kilian, and L. Salvail. On the (im)possibility of basing oblivious transfer and bit commitment on weakened security assumptions. In EUROCRYPT '99, volume 1592 of $L N C S$, pages 56-73. Springer, 1999.

14. Y. Dodis and S. Micali. Lower bounds for oblivious transfer reductions. In $E U$ ROCRYPT '99, volume 1592 of LNCS, pages 42-55, 1999.

15. J. Forster. A linear lower bound on the unbounded error probabilistic communication complexity. In 16th Conf. on Comput. Complexity, pp. 100-106, 2001.

16. O. Goldreich and R. Vainish. How to solve any protocol problem-an efficiency improvement. In CRYPTO '87, vol. 293 of LNCS, pages 73-86. Springer, 1988.

17. Y. Ishai, J. Kilian, K. Nissim, and E. Petrank. Extending oblivious transfers efficiently. In CRYPTO 2003, volume 2729 of $L N C S$, pages 145-161, Springer, 2003.

18. J. Kilian. Basing cryptography on oblivious transfer. In Proc. of the 20th Symp. on the Theory of Computing, pages 20-31, 1988.

19. J. Kilian. A general completeness theorem for two-party games. In Proc. of the 23th Symp. on the Theory of Computing, pages 553-560, 1991.

20. J. Kilian. More general completeness theorems for two-party games. In Proc. of the 32nd Symp. on the Theory of Computing, pages 316-324, 2000. 
21. J. Kilian, E. Kushilevitz, S. Micali, and R. Ostrovsky. Reducibility and completeness in private computations. SIAM J. on Computing, 28(4):1189-1208, 2000.

22. E. Kushilevitz. Privacy and communication complexity. SIAM J. on Discrete Mathematics, 5(2):273-284, 1992.

23. E. Kushilevitz and N. Nisan. Communication Complexity. Cambridge University Press, 1997.

24. U. Maurer. Information-theoretic cryptography. In CRYPTO '99, volume 1666 of LNCS, pages 47-64. Springer, 1999.

25. M. Naor and K. Nissim. Communication preserving protocols for secure function evaluation. In Proc. of the 33th Symp. on the Theory of Computing, 2001. 